\title{
Efficient fiber-laser pumped Ho:LuLiF 4 laser
}

\author{
J. W. Kim ${ }^{*}$, J. I. Mackenzie ${ }^{1}$, Daniela Parisi ${ }^{2}$, Stefano Veronesi ${ }^{2}$, Mauro Tonelli ${ }^{2}$, and \\ W. A. Clarkson ${ }^{1}$ \\ ${ }^{1}$ Optoelectronics Research Centre, University of Southampton, Highfield, Southampton SO17 1BJ, \\ United Kingdom. \\ ${ }^{2}$ National Enterprise for Nano Science and Technology-INFM - CNR, Dipartimento di Fisica \\ Università di Pisa, Largo B. Pontecorvo 3, 56127 Pisa, Italy. \\ jwk@orc.soton.ac.uk
}

\begin{abstract}
:
We present results for an efficient $\mathrm{Ho}: \mathrm{LuLiF}_{4}$ laser in-band pumped by a cladding-pumped Tm-doped silica fiber laser. The polarized, tunable Tm-doped silica fiber-laser operates at $1938 \mathrm{~nm}$, ideally suited for in-band pumping the $40 \mathrm{~mm}$ long weakly-doped $\left(0.25\right.$ at.\%) $\mathrm{Ho}_{\mathrm{LuLiF}}$ crystal. Using a simple laser resonator a maximum output power of $5.1 \mathrm{~W}$ was achieved at a wavelength of $2066 \mathrm{~nm}$ for $8.0 \mathrm{~W}$ of absorbed pump power, when using an output coupling mirror with $20 \%$ transmission, corresponding to a slope efficiency of $70 \%$. At a higher cavity output coupling of $37 \%$, the lasing wavelength shifted to a higher gain peak at $2053 \mathrm{~nm}$, where a maximum output power of $5.4 \mathrm{~W}$ was obtained with a slope efficiency of $76 \%$. Beam quality was measured to be $\mathrm{M}^{2} \sim 1.1$ at the maximum output power for each resonator configuration. The spectroscopy, lifetime of the upper laser level, and the laser performance will be discussed in terms of future prospects for power scaling and further improvements in the laser efficiency.
\end{abstract}

Keywords: Diode-pumped; Solid-state lasers; Fiber-laser; Fiber-laser pumped; Ho:LuLiF

\section{INTRODUCTION}

Efficient solid-state lasers in the eye-safe 2-micron regime are required for a variety of applications including coherent LIDAR, health care, materials processing, and as a pump source for nonlinear frequency conversion into the mid infrared spectral regime. Holmium lasers [1-4] are often preferred over thulium lasers due to their longer operating laser wavelength, benefiting from lower atmospheric or materials absorption. Additionally, the holmium ${ }^{5} \mathrm{I}_{8}$ metastable level usually has a longer lifetime than the thulium ${ }^{3} \mathrm{~F}_{4}$ level and a stronger emission cross section; consequently Ho-doped active media have an order-of-magnitude lower saturation intensity and therefore offer better characteristics for Qswitched operation and the potential for generating very high pulse energies [5, 6]. Unfortunately however, commercially available laser diodes suitable for direct pumping of holmium ions have much lower brightness and efficiency in comparison to other standard diode-laser wavelengths, therefore alternate routes, such as thulium sensitizers [7,8] or Tm: laser in-band pumping of holmium [1,9, 10], are generally considered the best routes for achieving efficient laser operation at high average powers.

Ho:LuLiF 4 (Ho:LLF) a relatively new crystal, invented at the NASA laboratories [3], has several advantageous properties that make it interesting as a potential source for highly energetic laser pulses, such as; a long upper-state lifetime approaching $15 \mathrm{~ms}$ that is crucial for energy storage, a strong spectral anisotropy leading to polarized absorption and emission characteristics, and a negative thermal dependence of the refractive index $(\mathrm{dn} / \mathrm{dT})$ that compensates the positive thermal expansion coefficient and hence end-face bulging, thus resulting in weak overall thermal lensing. Moreover the LLF crystal also benefits from a closer match in its thermal expansion coefficient and conductivity (k) along each of the crystal axes, with respect to its isomorphs $\mathrm{YLiF}_{4}$ and $\mathrm{GdLiF}_{4}$ [11]. Notwithstanding, this gain medium suffers strong reabsorption at its lasing wavelengths due to weak crystal fields and hence Stark-level splitting of the energy levels, leading to a significant population in the terminal laser level in the ${ }^{5} \mathrm{I}_{7}$ ground-state manifold [11], and therefore requires a high radiance excitation source to enable efficient operation; making it ideally suited to the hybrid fiber-pumping-bulk configuration as described here.

\footnotetext{
Solid State Lasers and Amplifiers IV, and High-Power Lasers, edited by Thomas Graf, Jacob I. Mackenzie, Helena Jelinková, Gerhard G. Paulus, Vincent Bagnoud, Catherine Le Blanc, Proc. of SPIE Vol. 7721, $77210 \mathrm{~V} \cdot$ @ $2010 \mathrm{SPIE} \cdot \mathrm{CCC}$ code: 0277-786X/10/\$18 $\cdot$ doi: 10.1117/12.853806
} 
The fiber-bulk hybrid laser architecture is especially promising for Ho-doped laser materials, which have moderate absorption characteristics in the $1.9 \mu \mathrm{m}$ wavelength region, and are well suited to the Tm-doped fiber-laser and their excellent potential for very high average powers coupled with the advantage of wavelength agility with a narrowlinewidth and good beam quality [12]. Furthermore these attributes of the fiber pump source allow the use of long bulk crystal lengths with low doping concentration, thus minimising detrimental effects like energy transfer upconversion losses [13]. Furthermore, such high brightness pump sources enable the bulk laser to be operated at many times the laser threshold, leading to effective saturation of the reabsorption losses and hence the possibility to achieve the maximum permissible slope efficiency [14], especially when capitalising on the resonator design flexibility afforded by the neardiffraction-limited-pump.

Here we will present some of the optical properties of singly-doped Ho:LuLiF 4 and what we believe to be the first inband pumped Ho:LLF laser utilizing a Tm-doped silica fiber laser. Excellent conversion efficiency was observed with respect to the absorbed pump power for this study, where in continuous wave (CW) operation a maximum $5.4 \mathrm{~W}$ of output power at $2.1 \mu \mathrm{m}$ was obtained for $8.0 \mathrm{~W}$ of absorbed Tm: fiber laser input was demonstrated.

\section{METHODOLOGY}

\subsection{Ho:LLF crystal growth}

The sample under investigation was a $\mathrm{LiLuF}_{4}$ single crystal doped with a concentration of 0.25 at.\% $\mathrm{Ho}^{3+}$, i.e. an ion density of $36 \mu^{-3}$. LLF has a scheelite structure with lattice parameters $\mathrm{a}=5.167 \AA$ and $\mathrm{c}=10.735 \AA$ and symmetry group I41/a; where each unit cell contains four formula units. The $\mathrm{Ho}^{3+}$ dopant substitutes $\mathrm{Lu}^{3+}$ in S4 symmetry sites and therefore has a coordination number of 8 . Important for the crystal integrity is the match in ionic radii (1.16 $\AA$ ) between $\mathrm{Lu}$ and Ho in this medium [15].

The Ho:LLF boule was grown in the NEST laboratories, which are equipped with a home-made computer-controlled Czochralski furnace with conventional resistive heating and an optical measurement apparatus. The growth of the sample was performed in a high vacuum $\left(<10^{-7} \mathrm{mbar}\right)$ in order to avoid the $\mathrm{OH}^{-}$contamination, with a high-purity $(99.999 \%)$ argon atmosphere. The constituents of the crystal comprised high purity $(5 \mathrm{~N}) \mathrm{LiF}$ and $\mathrm{LuF}_{3}$ and $\mathrm{HoF}_{3}$ powders as raw materials provided by AC Materials (Tarpon Springs, Flo., USA). The average size of the as LLF boule grown in the vitreous carbon crucible was about $10 \mathrm{~mm}$ in diameter by $70 \mathrm{~mm}$ in length, free from obvious cracks or defects. The laser crystal sample was cleaved from such a boule to dimensions of $5.4 \times 5.3 \times 40 \mathrm{~mm}^{3}$, with the crystallographic c-axis orthogonal to the longest edge. The end faces where then polished plane and parallel and anti-reflection coated to cover the wavelength range from 1900-2100 nm.

\subsection{Optical characterization}

A room temperature life time measurement was performed by exciting a smaller crystal sample with a $\mathrm{BaY}_{2} \mathrm{~F}_{8}: \mathrm{Tm}^{3+}$ laser emitting at $1932 \mathrm{~nm}$, which was developed for such tasks and described previously in [16]. This $50 \mathrm{~mW}$ CW pump

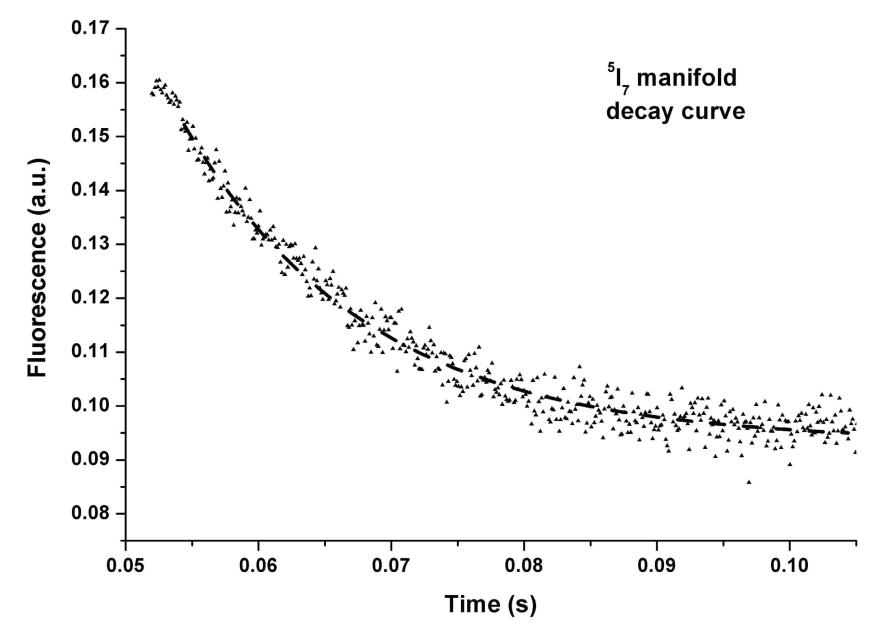

Figure 1: Lifetime measurement of the directly-excited ${ }^{5} \mathrm{I}_{7}$ manifold. 
source was modulated with an optical chopper and the beam focused using a $10 \mathrm{~cm}$ focal length lens to a spot waist size of $500 \mu \mathrm{m}$ in a short sample of $\sim 1 \mathrm{~mm}$ length, so to ensure the energy density was as constant as possible in the probed region and to reduce any undesired effects, such radiation trapping. Furthermore with appropriate attenuation the effects of up-conversion that could affect the life time of the upper laser manifold could also be mitigated. The fluorescence was collected perpendicular to the excitation beam propagation direction, to facilitate ease of discrimination between the signal and pump. The signal was acquired using an InSb detector, cooled to the liquid nitrogen temperature. As it can be seen in figure 1 the decay curve shows a single exponential behavior and the life time value of the ${ }^{5} \mathrm{I}_{7}$ manifold was $14.1 \pm 0.5 \mathrm{~ms}$, in good agreement with that published in the literature [17].

Room temperature polarized absorption measurements were performed using a CARY 500 spectrophotometer in the range $250-2200 \mathrm{~nm}$ with a resolution of $0.6 \mathrm{~nm}$ in the UV region and of $1.5 \mathrm{~nm}$ in the IR region, in order to check the absorption lines of the $\mathrm{Ho}^{3+}$ ion and also to verify the absence of lines belonging to other impurities. Presented in figure 2 is a detailed spectrum over the wavelength range of 1850 to $2200 \mathrm{~nm}$, which corresponds to the transitions between the ${ }^{5} \mathrm{I}_{8} \rightarrow{ }^{5} \mathrm{I}_{7}$ manifolds. We performed these measurements on the AR-coated crystal sample used in our laser experiments, with the light polarized in each arm of the spectrophotometer according to the respective crystal axes. The resolution was set to $0.5 \mathrm{~nm}$ along with a suitable averaging time in order to optimize the signal to noise ratio. In figure 2 we show the recorded absorption spectra for both the $\pi$ and $\sigma$ polarizations. The most intense absorption peak is $\pi$ polarized and lies at $1937.7 \mathrm{~nm}$ with a cross section $\sigma_{\mathrm{abs}}=0.85 \mathrm{pm}^{2}$ corresponding to a small-signal extinction coefficient in our crystal of $\alpha_{\mathrm{p}}=0.3 \mathrm{~cm}^{-1}$; for the $\sigma$ polarization the highest absorption cross section is $\sigma_{\mathrm{abs}}=0.45 \mathrm{pm}^{2}$, i.e. $\alpha=0.16 \mathrm{~cm}^{-1}$, at $1943.5 \mathrm{~nm}$.

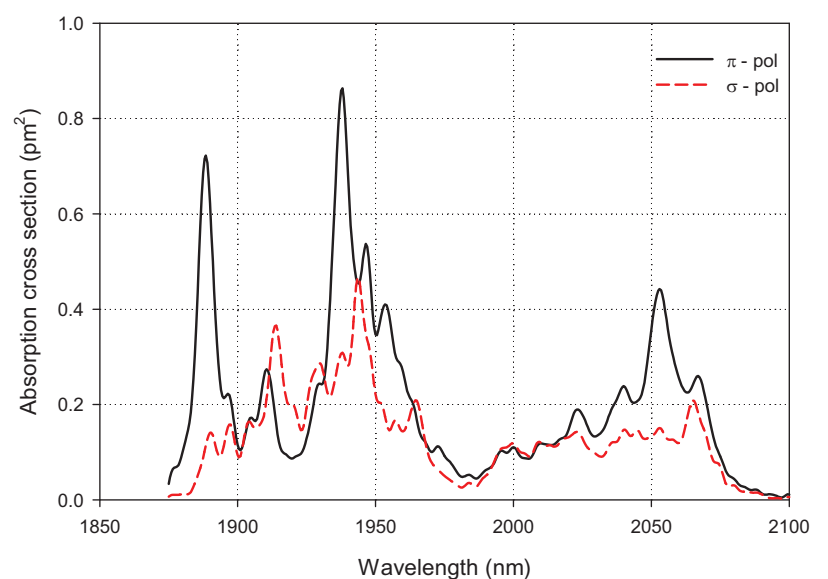

(a)

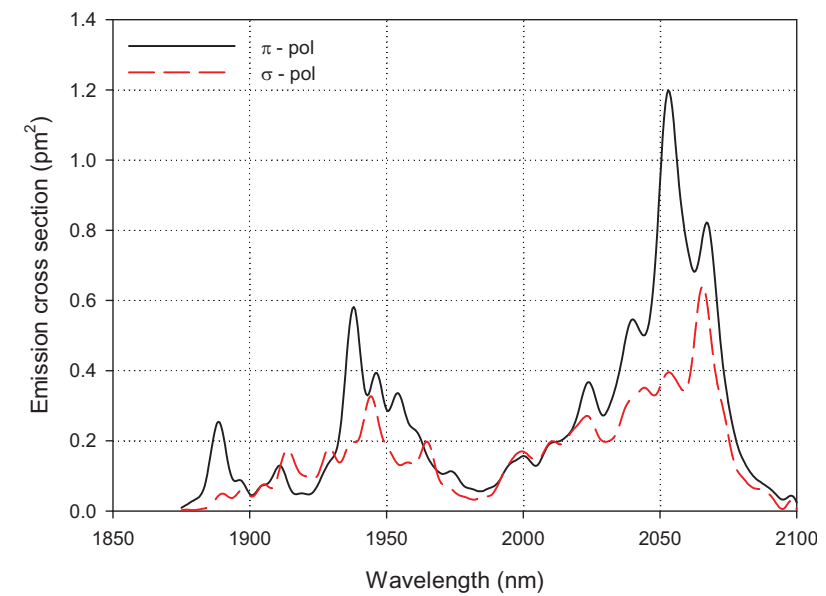

(b)

Figure 2: (a) Measured absorption cross section and (b) the emission cross section calculated by the reciprocity method from the data in (a). 


\subsection{Laser experiment set-up}

An end-pumping configuration as shown in figure 3 was used in our Ho:LLF laser experiments. A Tm: fiber pump laser was constructed for this experiment comprising a $\sim 4 \mathrm{~m}$ length of a commercially available Tm-doped polarization maintaining (PM) fiber, with a $25 \mu \mathrm{m}$ diameter - $0.1 \mathrm{NA}$ core, and a $0.4 \mathrm{NA}$ cladding with a diameter of $400 \mu \mathrm{m}$. This pump laser was setup to operate at a wavelength coincident with the strongest Ho:LLF absorption peak at $1938 \mathrm{~nm}$, by employing an external cavity consisting of a collimating lens $(\mathrm{f}=100 \mathrm{~mm})$, a half-wave plate, polarizer and a replica diffraction grating $(600$ lines $/ \mathrm{mm})$ in the Littrow arrangement. The output beams of two spatially- and polarizationmultiplexed $795 \mathrm{~nm}$ diode-stacks were again split in the slow axis to generate two beams of roughly equal power and beam quality characteristics allowing pumping of the Tm: fiber from both ends. This Tm: fiber laser arrangement yielded $25 \mathrm{~W}$ of a linearly-polarized output at $1938 \mathrm{~nm}$ in a beam with an $\mathrm{M}^{2}<1.8$ for $93 \mathrm{~W}$ of launched pump power. The Tm: fiber laser output was collimated, and another half-wave plate and polarizer were used to improve the final polarization extinction ratio, whilst also providing the ability to align the electric field to the c-axis of the Ho:LLF crystal, i.e. the strongest absorption feature in figure 2 . One of the beam steering mirrors used to redirect the Tm: fiber laser output had a $10 \%$ transmission to allow monitoring of the incident pump power at all times during the experiment.

The Ho:LLF laser resonator was configured as a "V-cavity" with three-mirrors and an intra-cavity lens, comprising a plane pump input-coupler (IC) mirror with high reflectivity (> 99.8\%) for the wavelength range 2050-2150 $\mathrm{nm}$ and high transmission (>95\%) at the $1938 \mathrm{~nm}$ pump wavelength, a $\mathrm{f}=100 \mathrm{~mm}$ plano-convex lens, a fold mirror (DM) with the same reflection/transmission characteristics as the IC, and a plane output coupler. In this way we could measure the unabsorbed pump power, and with appropriate isolation of the pump source, it would be possible retro-reflect this light to achieve double-pass of the pump light through the crystal. A suitable isolator was not available at the time of the experiment and hence all the results are described in terms of absorbed power as determined from the measured unabsorbed pump power after a single pass of the laser crystal. Several output coupling mirrors with transmittance $\left(\mathrm{T}_{\mathrm{oc}}\right)$ of $3 \%, 10 \%, 20 \%$ and $37 \%$ were investigated, leading to different levels of ground state bleaching and actual percentage of absorbed pump power. The $40 \mathrm{~mm}$ long Ho:LLF crystal was mounted in a water-cooled copper heat-sink maintained at a temperature of $17^{\circ} \mathrm{C}$ and positioned in close proximity to the plane mirror (IC). The single-pass small-signal absorption efficiency at $1938 \mathrm{~nm}$ was measured to be $\sim 70 \%$. The physical length of the resonator was $\sim 300 \mathrm{~mm}$ resulting in a calculated $\mathrm{TEM}_{00}$ waist radius of $\sim 200 \mu \mathrm{m}$ and, hence, the pump beam from the Tm: fiber laser was conditioned to have the same beam waist size in the Ho:LLF crystal.

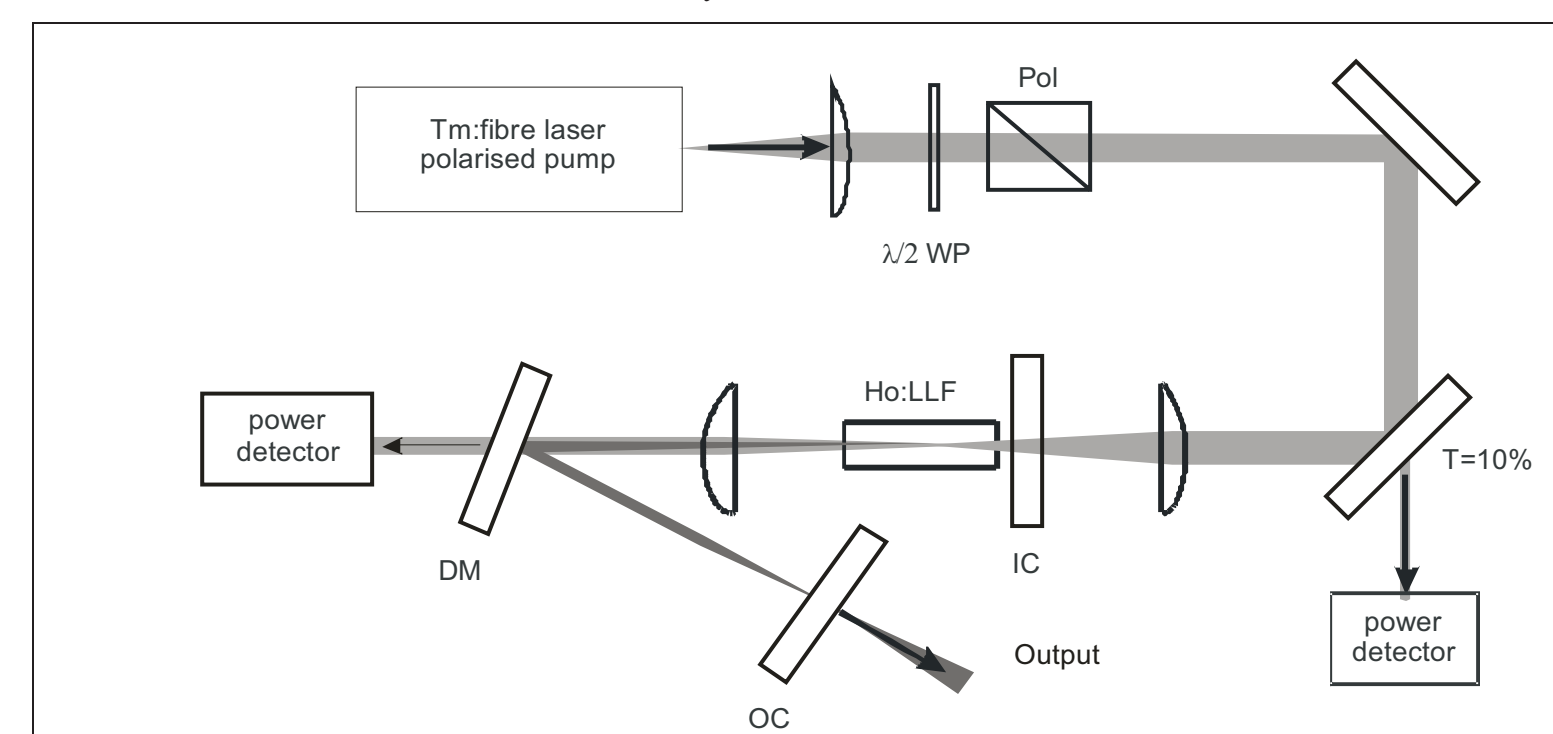

Figure 3: Set up for the Ho:LLF laser resonator 


\section{RESULTS AND DISCUSSION}

For the resonator configuration illustrated in figure 3, and three of the four different output coupling mirrors used, i.e. $\mathrm{T}_{\mathrm{oc}}=3 \%, 10 \%$ and $20 \%$, the laser operated at a wavelength of $2066 \mathrm{~nm}$ with a measured output power as a function of absorbed pump power as shown in figure. 4. The slope efficiency increases with output coupler transmission, which is attributed to saturation of the strong re-absorption losses, calculated to be nearly an order of magnitude higher than the round trip cavity loss at threshold for the $10 \% \mathrm{~T}_{\text {oc }}$ mirror (i.e. B 8 using the notation of Risk [14]). With an output coupler of $20 \% \mathrm{~T}_{\mathrm{oc}}$, the Ho:LLF laser produced $5.1 \mathrm{~W}$ of output for $8.0 \mathrm{~W}$ of absorbed pump, giving a slope efficiency of $70 \%$ with respect to the absorbed power. Threshold was determined as the absorbed pump power required for the onset of relaxation oscillations measured using an InGaAs photodetector and oscilloscope, and found to be $420 \mathrm{~mW}, 440 \mathrm{~mW}$ and $470 \mathrm{~mW}$ for $3 \%, 10 \%$, and $20 \% \mathrm{~T}_{\mathrm{oc}}$ output coupling mirrors respectively. The output was $\pi$-polarized and the beam quality measured to be $\mathrm{M}^{2} \sim 1.1$ at the maximum output power.

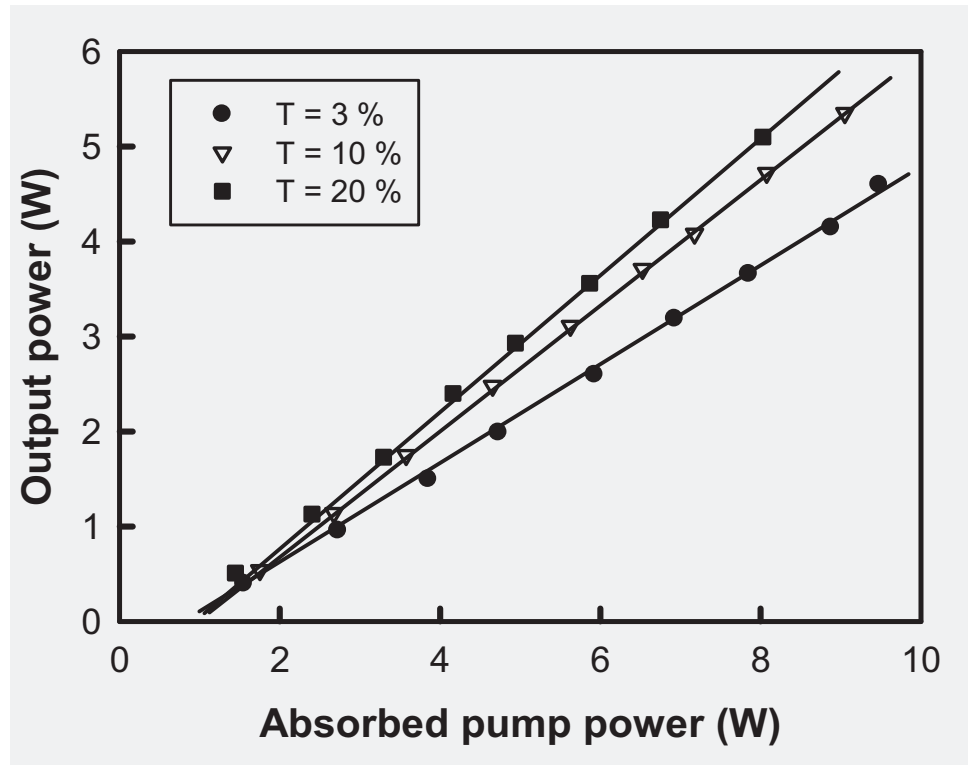

Figure 4: Output power versus the absorbed pump power for $(\bullet) 3 \% \mathrm{~T},(\nabla) 10 \% \mathrm{~T}$, and $(\boldsymbol{\square}) 20 \% \mathrm{~T}$ output coupling mirrors.

Significant ground state depletion was observed with increasing cavity loss, as can be seen with the decreasing absorption efficiency in figure 4 (noting that nominally the same pumping powers were used in each case), reducing to $\sim 50 \%, \sim 48 \%, \sim 43 \%$ under lasing conditions for the output couplers $\mathrm{T}_{\mathrm{oc}}=3 \%, 10 \%$ and $20 \%$, respectively. This behavior is consistent with the moderate inversion required to achieve transparency of the holmium doped crystal at the lasing wavelength and sufficient round trip gain to overcome the unsaturable cavity losses, noting that at room temperature transparency is achieved at $2066 \mathrm{~nm}$ when $21 \%$ of the $\mathrm{Ho}^{3+}$ ions are excited to the ${ }^{5} \mathrm{I}_{8}$ energy level.

When using the $37 \% \mathrm{~T}_{\mathrm{oc}}$ output coupler however, the Ho:LLF laser shifted its operating wavelength to $2053 \mathrm{~nm}$, where the effective gain at this wavelength exceeds that at $2066 \mathrm{~nm}$ due to the higher inversion levels required to reach threshold. Using the measured absorption spectrum and the calculated emission cross section, the effective gain as a function of wavelength was determined for the various output coupling mirrors reflectivities, such that the steady state round-trip gain, equivalent to the round trip loss, is achieved assuming the same small cavity losses ( $\sim 1 \%$ round trip). Figure 5 illustrates the Ho:LLF gain coefficient as a function of wavelength, calculated for the various output couplers used in our experiments. We only show the curves for the $\pi$-polarization, as this is the dominant polarization state under the given conditions. It is shown that for the smaller output coupling mirror transmissions, $\mathrm{T}_{\mathrm{oc}}=3 \%, 10 \%$ and $20 \%$, which corresponds to population inversion levels, $\beta=22 \%, 24 \%$ and $28 \%$ respectively, the gain is higher for the $2066 \mathrm{~nm}$ transition, whereas for the $37 \% \mathrm{~T}_{\mathrm{oc}}$ mirror the $2053 \mathrm{~nm}$ line dominates with a calculated $\beta=34 \%$. In addition we estimate the cavity output coupling at which the gain for the $2053 \mathrm{~nm}$ lines equals that of the $2066 \mathrm{~nm}$ for small cavity losses is $\sim 24 \%$ where $\beta \sim 30 \%$. These gain dynamics are important when considering operating in the Q-switched 
regime where typically a high transmission output coupler is necessary, according to the damage threshold limits of the intra-cavity optics, and which will enforce laser operation at the shorter $2053 \mathrm{~nm}$ wavelength, where there is a higher initial reabsorption loss. At the same time as observed in our experiments and as can be seen in figure 5, ground state bleaching does becomes significant for the $1938 \mathrm{~nm}$ pump wavelength with increasing output coupling. With these factors in mind it is important that efficient $\mathrm{CW}$ operation is possible with higher cavity output coupling as demonstrated in figure 6, the resulting laser output power at $2053 \mathrm{~nm}$ with the $37 \% \mathrm{~T}_{\mathrm{oc}}$ mirror. The maximum output power was $5.4 \mathrm{~W}$ for $8.0 \mathrm{~W}$ of absorbed pump power with a slope efficiency of $76 \%$. Threshold pump power was measured, again by observation of relaxation oscillations, to be $550 \mathrm{~mW}$.

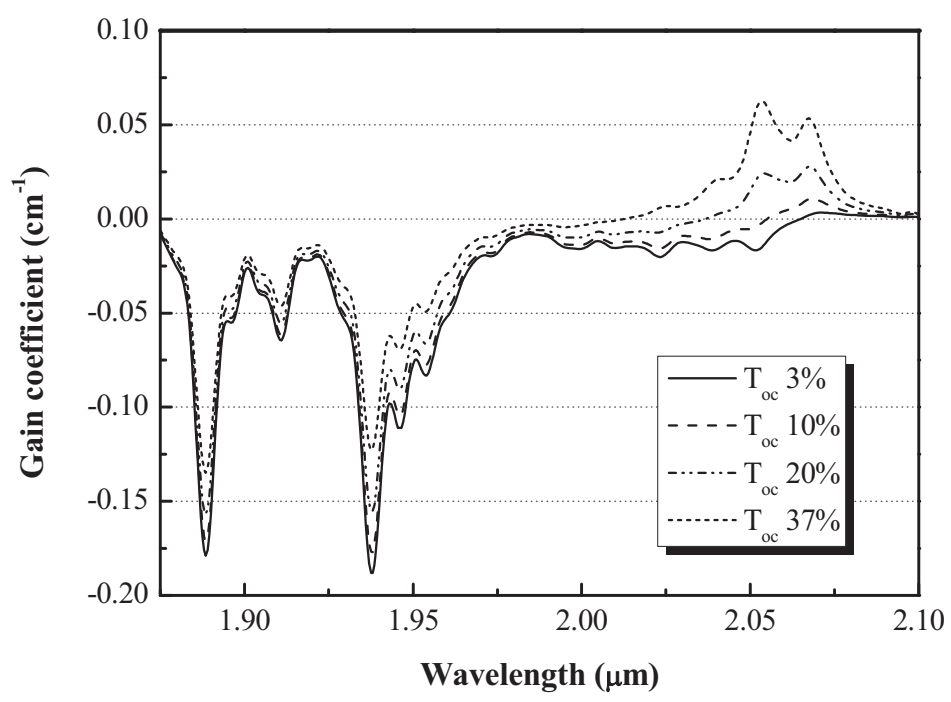

Figure 5: Gain coefficient as a function of wavelength for various output coupling mirrors.

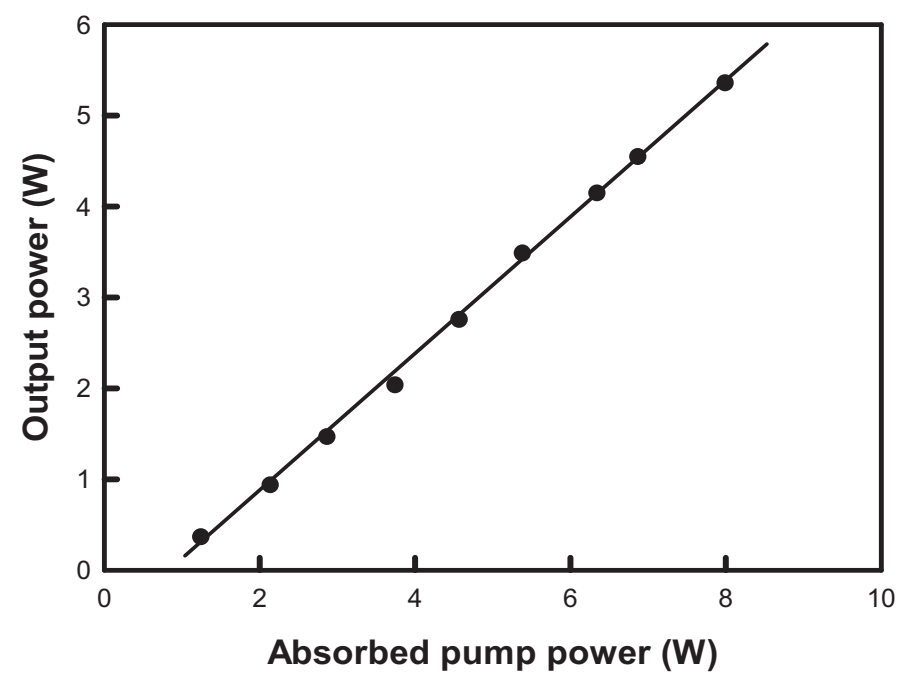

Figure 6: Output power at $2053 \mathrm{~nm}$ for a $37 \% \mathrm{~T}_{\mathrm{oc}}$ output coupler mirror.

\section{CONCLUSIONS}

We have demonstrated that singly-doped Ho:LLF appears to be an excellent candidate for in-band pumped 2.1 micron operation, especially with high brightness Tm: fiber laser pump sources. The hybrid-laser approach is also particularly promising for a Q-switched mode of operation due to the anticipated long energy storage lifetime and low saturation 
fluence/intensity [11]. The significant ground state population of the strongest laser transitions will mean the effects of detrimental thermal loading of the crystal through reabsorption of fluorescent power and ETU processes will need careful evaluation, particularly when operating in a pulsed mode, where the Ho:LLF laser is more likely to operate at wavelength of $2053 \mathrm{~nm}$, which is especially susceptible to laser performance degradation (i.e. power, beam quality, and extractable energy) [18]. The absorption and emission properties of this singly-doped Ho:LLF for the laser transition between the ground and first excited state manifold were measured and reported here, with a peak absorption around $1938 \mathrm{~nm}$ with a cross section of $\sigma_{\mathrm{abs}}=0.85 \mathrm{pm}^{2}$ for $\pi$-polarized light. The lifetime of the 2.1 micron ${ }^{5} \mathrm{I}_{7}$ upper laser level was measured to be $14.1+/-0.5 \mathrm{~ms}$ with no evidence of excited state energy transfer processes. Furthermore ETU appears to be negligible in our experimental configuration as might be expected with such a low holmium dopingconcentration. This highlights the possibility of employing higher doping levels with scope to further optimize the absorption efficiency and crystal length and developing power scaling strategies for this gain medium [19].

In conclusion, we have investigated the spectroscopic properties of Ho:LLF and its continuous-wave laser performance in the 2.1 micron wavelength regime, when in-band pumped by a polarized Tm:fiber laser operating at $1.94 \mu \mathrm{m}$. Wavelength selection characteristics at threshold and the effect of moderate ground state bleaching have been considered, with the conclusion that the Ho:LLF laser investigated will operate at $2053 \mathrm{~nm}$ for cavity output coupling transmissions in excess of $24 \%$. In our study of a simple three-mirror resonator Ho:LLF laser we observed very efficient operation, where for a lasing wavelength of $2066 \mathrm{~nm}$ the best slope efficiency achieved was 70\%, and at $2053 \mathrm{~nm}$ it was $76 \%$. In these experiments we were limited by the available pump power from our customized Tm: fiber laser, with further optimization of both the pump and Ho:LLF crystal, e.g. crystal length and holmium doping concentration, 2.1 micron laser power levels well in excess of the $\sim 5 \mathrm{~W}$ levels shown here are feasible. In the event of increasing the average output power suitable strategies for minimizing detrimental thermal-lensing and ETU effects, associated with high inversion densities, will need to be investigated further. We have demonstrated a Tm:fiber-laser pumped Ho:LLF laser with excellent conversion efficiency, further power scaling of this low-quantum defect transition look particularly attractive, in addition it is expected that this active medium will be an excellent candidate for generating high pulse energies in the 2.1 micron regime.

\section{REFERENCES}

[1] P. A. Budni, M. L. Lemons, J. R. Mosto et al., "High-power/high-brightness diode-pumped 1.9 4 m Thulium and resonantly pumped $2.1 \mu \mathrm{m}$ Holmium lasers," IEEE Journal of Selected Topics in Quantum Electronics, 6(4), 629-635 (2000).

[2] N. P. Barnes, B. M. Walsh, and E. D. Filer, "Ho : Ho upconversion: applications to Ho lasers," Journal of the Optical Society of America B-Optical Physics, 20(6), 1212-1219 (2003).

[3] B. M. Walsh, "Review of Tm and Ho materials; spectroscopy and lasers," Laser Physics, 19(4), 855-866 (2009).

[4] X. Yu, F. Chen, J. Gao et al., "Diode-laser-pumped high efficiency continuous-wave operation at $912 \mathrm{~nm}$ laser in Nd:GdVO 4 crystal," Laser Physics Letters, 6(1), 34-37 (2009).

[5] R. Brinkmann, D. Theisen, T. Brendel et al., "Single-pulse 30-J holmium laser for myocardial revascularization - A study on ablation dynamics in comparison to $\mathrm{CO}_{2}$ laser-TMR," IEEE Journal of Selected Topics in Quantum Electronics, 5(4), 969-980 (1999).

[6] J. R. Yu, B. C. Trieu, E. A. Modlin et al., "1 J/pulse Q-switched 2 mu m solid-state laser,” Optics Letters, 31(4), 462-464 (2006).

[7] T. Y. Fan, G. Huber, R. L. Byer et al., "Spectroscopy and Diode Laser-Pumped Operation of Tm, Ho:YAG," IEEE Journal of Quantum Electronics, 24(6), 924-933 (1988).

[8] V. Sudesh, K. Asai, K. Shimamura et al., "Pulsed laser action in Tm,Ho:LuLiF 4 and Tm,Ho:YLiF 4 crystals using a novel quasi-end-pumping technique,” IEEE Journal of Quantum Electronics, 38(8), 1102-1109 (2002).

[9] D. Y. Shen, A. Abdolvand, L. J. Cooper et al., "Efficient Ho:YAG laser pumped by a cladding-pumped tunable Tm : silica-fiber laser," Applied Physics B-Lasers and Optics, 79(5), 559-561 (2004).

[10] S. So, J. I. Mackenzie, D. P. Shepherd et al., "Intra-cavity side-pumped Ho:YAG laser,” Optics Express, 14(22), 10481-10487 (2006).

[11] B. M. Walsh, N. P. Barnes, M. Petros et al., "Spectroscopy and modeling of solid state lanthanide lasers: Application to trivalent $\mathrm{Tm}^{3+}$ and $\mathrm{Ho}^{3+}$ in $\mathrm{YLiF}_{4}$ and $\mathrm{LuLiF}_{4}$," Journal of Applied Physics, 95(7), 3255-3271 (2004). 
[12] P. F. Moulton, G. A. Rines, E. V. Slobodtchikov et al., "Tm-Doped Fiber Lasers: Fundamentals and Power Scaling," IEEE Journal of Selected Topics in Quantum Electronics, 15(1), 85-92 (2009).

[13] J. W. Kim, J. I. Mackenzie, and W. A. Clarkson, "Influence of energy-transfer-upconversion on threshold pump power in quasi-three-level solid-state lasers," Optics Express, 17(14), 11935-11943 (2009).

[14] W. P. Risk, "Modeling of Longitudinally Pumped Solid-State Lasers Exhibiting Reabsorption Losses," Journal of the Optical Society of America B-Optical Physics, 5(7), 1412-1423 (1988).

[15] A. A. Kaminskii, [Laser Crystals: Their Physics and Properties.] Springer-Verlag, New York(1990).

[16] F. Cornacchia, D. Parisi, C. Bernardini et al., "Efficient, diode-pumped $\operatorname{Tm}^{3+}: \mathrm{BaY}_{2}\left(\mathrm{~F}_{8}\right)$ vibronic laser," Optics Express, 12(9), 1982-1989 (2004).

[17] B. M. Walsh, G. W. Grew, and N. P. Barnes, "Energy levels and intensity parameters of $\mathrm{Ho}^{3+}$ ions in $\mathrm{GdLiF}_{4}$, $\mathrm{YLiF}_{4}$ and $\mathrm{LuLiF}_{4}$," Journal of Physics-Condensed Matter, 17(48), 7643-7665 (2005).

[18] J. W. Kim, D. Y. Shen, J. K. Sahu et al., "High-power in-band pumped Er:YAG laser at 1617 nm," Optics Express, 16(8), 5807-5812 (2008).

[19] S. So, J. I. Mackenzie, D. P. Shepherd et al., "A power-scaling strategy for longitudinally diode-pumped Tm:YLF lasers,” Applied Physics B-Lasers and Optics, 84(3), 389-393 (2006). 